\title{
Die regionalen Rates of Return der öffentlichen Hochschulfinanzierung*
}

\author{
Die Regional Rates of Return \\ on Public University Financing
}

\begin{abstract}
Kurzfassung
Der vorliegende Artikel untersucht die kurzfristigen regionalökonomischen Wirkungen von Hochschulen. Die dabei verwandte Methodik unterscheidet sich wesentlich von bisherigen Studien, denn sie stellt auf die von den Hochschulen zusätzlich attrahierten Mittel ab und setzt deren Effekte ins Verhältnis zur Grundfinanzierung durch das Land. Die so berechneten Verhältniszahlen nennen wir die regionalen Rates of Return der Hochschulfinanzierung. Exemplarisch werden die Effekte am Beispiel der Otto-von-Guericke-Universität Magdeburg und der Hochschule Magdeburg-Stendal (FH) berechnet. Die Ergebnisse zeigen, dass die Finanzierung von Hochschulen wirtschafts- und arbeitsmarktpolitisch besonders effektiv ist und dass Kürzungen im Hochschulbereich auch kurzfristig erhebliche Opportunitätskosten verursachen.
\end{abstract}

\begin{abstract}
This article examines the short-term regional economic effects of universities. The methodology used differs substantially from previous studies as it focuses on the additional resources attracted to the region by the establishment of universities. The effects of the attracted resources are therefore related to the basic financing provided by the federal state (Land). We call these relations the regional rates of return on university financing. We demonstrate the regional rates of return using the experience of the Otto-von-GuerickeUniversity Magdeburg and the college of higher education Magdeburg-Stendal as examples. The results indicate that the financing of universities is an effective means to create both jobs and economic prosperity. In turn, the reduction of public funding for universities causes substantial opportunity costs for the region, even in the short term.
\end{abstract}

\section{Einleitung}

Die Finanzierung von Hochschulen durch die Länder ist häufig mit der Erwartung verknüpft, dass regionalökonomische Wirkungen von ihnen ausgehen. Obwohl die Existenz solcher Wirkungen unbestritten ist, herrscht oftmals Unklarheit über deren Art und Ausmaß. Angesicht knapper Ressourcen stellt sich den Ländern zunehmend die Frage, welche Effekte die Hochschulen auf die umliegenden Regionen tatsächlich haben und ob diese Effekte den erheblichen Mitteleinsatz rechtfertigen. Diese Frage stellt sich nicht nur der Politik, die letztlich die Hochschulressourcen bereitstellen muss, sondern zunehmend auch den Hochschulen selbst. Die wachsende Finanznot der
Bundesländer hat in den vergangenen Jahren immer wieder zu erheblichen Sparmaßnahmen im Hochschulbereich geführt. Dies verwundert auf den ersten Blick, denn der Anteil der Ausgaben für das Hochschulwesen (gemessen am BIP) ist im OECD-Vergleich in Deutschland relativ gering. Gleichzeitig gehört es zum Fundus der gesicherten Erkenntnisse aller gesellschaftlichen Gruppen, dass Deutschland existenziell auf hochwertige Forschung und Lehre angewiesen ist. Daraus sollte eigentlich eine Stärkung der Hochschulen folgen, aber tatsächlich ist das Gegenteil der Fall. Ein Grund dafür dürfte in der Tatsache zu finden sein, dass die Hochschulfinanzierung Ländersache ist und 
die Länder sich einem erheblichen Spardruck ausgesetzt sehen, dem zu folgen deshalb schwer fällt, weil die Bundesländer nur in wenigen Bereichen autonom über ihre Ausgaben entscheiden können. Die Hochschulen sind ein solcher Bereich und geraten deshalb fast zwangsläufig in das Visier der Finanzminister. Dazu kommt, dass Einsparungen bei den Hochschulen zumindest kurzfristig scheinbar folgenlos bleiben. Positive Effekte erwartet man von Universitäten und Fachhochschulen eher in der langen Frist. Die Folge ist, dass Hochschulen ein Sparpotential darstellen, das den Wählern leicht vermittelt werden kann, weil Einsparungen nicht zu unmittelbar spürbaren Einschränkungen der Versorgung mit öffentlich bereitgestellten Gütern führen - ganz anders als beispielsweise bei Theatern, Kindertagesstätten und ähnlichen Einrichtungen.

Vor diesem Hintergrund gewinnt die Frage, welche $u n$ mittelbaren, direkten und kurzfristige Effekte eine Hochschule für die sie beherbergende Region hat, erheblich an Bedeutung - sowohl für die Politik, die über Sparmaßnahmen zu entscheiden hat, als auch für die Hochschulen, die um ihre Legitimation kämpfen. Es kann deshalb nicht überraschen, dass die regionalökonomische Bedeutung von Hochschulen in der Vergangenheit vielfach empirisch untersucht worden ist. Die jüngsten Studien beziehen sich auf die Universität Bielefeld (Niermann 1996), die Wissenschaftseinrichtungen in Hamburg (Clermont 1997), die Wissenschaftseinrichtungen in Bremen (Pfähler et al. 1999), die Universität Potsdam (Gloede et al. 1999) und die Universität Gesamthochschule Kassel (Blume/Fromm 2000). Alle diese Untersuchungen zeigen sämtliche Nachfrageeffekte, die regional im Laufe eines Jahres aufgrund der Einrichtungen entstehen, d.h. sie untersuchen die Wirkungen, die von den Ausgaben des Landes für eine Hochschule ausgehen.

Blume und Fromm (1999, S. 419) merken an, „dass auch eine alternative Mittelverwendung denkbar wäre. Große öffentliche Infrastruktureinrichtungen (wie z.B. ein Gefängnis oder ein Theater) weisen von der Seite der Leistungserstellung im Hinblick auf die Personal-, Sach- und Bauausgaben sicherlich ähnliche Wirkungen wie eine Hochschule auf. Unterschiede ergeben sich im Wesentlichen durch die Fähigkeit der Hochschule, Studenten und Drittmittel zu attrahieren (...)“. Dieser Punkt ist nach unserer Auffassung von zentraler Bedeutung. Wenn es um die Entscheidung geht, welche öffentlichen Projekte zum Gegenstand von Sparentscheidungen werden sollen, dann bedarf es einer Differentialbetrachtung, die die tatsächlichen Opportunitätskosten einer Sparentscheidung aufzeigt. Etwas plakativ formuliert: Es gilt herauszufinden, ob es einen Unterschied macht, ob ein Land in eine Hochschule investiert oder in den Bau einer Kantine. Dazu müssen die ökonomischen Effekte identifiziert werden, die nur deshalb zustande kommen, weil Ressourcen in Hochschulen und nicht in andere Projekte gesteckt werden. Diesen Punkt lassen die bisherigen Studien unbeantwortet. Im Unterschied dazu ist der vorliegende Beitrag durch folgendes Vorgehen gekennzeichnet:

\section{(1)}

Wir wählen einen methodischen Ansatz, der sich wesentlich von bisherigen Studien unterscheidet, indem ausschließlich die spezifischen Effekte untersucht werden, die sich für das Land zusätzlich infolge der Hochschulfinanzierung ergeben. Dabei wird ausschließlich auf kurzfristige Effekte abgestellt, die nur deshalb entstehen, weil die Hochschulen existieren und produzieren. Alle Effekte, die durch die Produktionsergebnisse einer Hochschule (Ausbildungs- und Forschungsleistungen, also Vermehrung von Humankapital und Wissen) entstehen, werden nicht berücksichtigt. Die zusätzlichen Regionaleffekte werden sodann ins Verhältnis zur Grundfinanzierung durch das Land gesetzt. Die so berechneten Verhältniszahlen nennen wir die regionalen Rates of Return der Hochschulfinanzierung. Mit den regionalen Rates of Return schlagen wir eine Berechnungsmethode für die Regionaleffekte von Hochschulen vor, die mehrere Vorteile bietet: Erstens liefert sie die gesamte gewonnene Information in konzentrierten Kennzahlen, die plastisch veranschaulichen, welche zusätzlichen Effekte die Hochschule einer Region zu geben vermag. Zweitens ermöglichen die regionalen Rates of Return die Identifizierung des spezifischen Vorteils der Hochschulfinanzierung und damit einen Vergleich zu alternativen Investitionsobjekten. Drittens können sie auch zum Vergleich von Hochschuleinrichtungen selber verwandt werden, um ihre Wirksamkeit aus regionalwirtschaftlichen Aspekten vergleichen zu können.

(2)

Wir untersuchen eine Universität und eine Fachhochschule gleichzeitig in einer Region und mit einer einheitlichen Methode, sodass strukturelle Unterschiede erkennbar werden, ohne dass räumliche oder politische Differenzen die Ergebnisse verzerren.

\section{(3)}

Wir wählen einen Betrachtungszeitraum über sechs Jahre hinweg. Im Gegensatz zu anderen Studien, die eine Momentaufnahme liefern, können dadurch verzerrende Einmaleffekte erkannt werden. Darüber hinaus werden Entwicklungstrends deutlich.

Unser Ansatz folgt der Einsicht, dass Universitäten und Fachhochschulen mit ihren Ausbildungs- und Forschungsleistungen letztlich handelbare Güter produzieren. Die Produktion solcher Güter führt normaler- 
weise zu Ressourcenzuflüssen, die aus dem Handel resultieren. Hochschulen geben ihre Produkte jedoch zum Preis Null ab, weil sie auf die Erhebung von Studiengebühren verzichten und auch Forschungsresultate in aller Regel ohne Entgelt zur Verfügung stellen. Dennoch löst der Handel mit den Hochschulprodukten Ressourcenflüsse in die Hochschulregion aus und genau diese gilt es zu identifizieren und zu quantifizieren. Dass der Einsatz der von den Hochschulen produzierten Güter in der Region ebenfalls wertschöpfend wirkt, soll dabei nicht vergessen werden, ist aber nicht Gegenstand dieser Untersuchung.

Der Artikel ist wie folgt organisiert: Abschnitt 2 beschreibt das methodische Vorgehen der Untersuchung. Abschnitt 3 zeigt die regionalökonomischen Effekte am Beispiel der Otto-von-Guericke-Universität Magdeburg und der Hochschule Magdeburg-Stendal (FH). In Abschnitt 4 werden die regionalökonomischen Effekte ins Verhältnis zu den Aufwendungen des Landes bei der Finanzierung der Hochschulen gesetzt und die regionalen Rates of Return ermittelt. Abschließend wird in Abschnitt 5 ein Fazit gezogen.

\section{Methodisches Vorgehen}

Ziel der vorliegenden Untersuchung ist die Bemessung der regionalen Rates of Return der Hochschulfinanzierung als Verhältnis von direkten Effekten zur Grundfinanzierung der Hochschulen. Als direkte Effekte der Hochschulfinanzierung gelten dabei alle regionalökonomischen Wirkungen, die auf reale Ressourcenzuflüsse von außerhalb der Hochschulregion zurückgehen. Sie kommen der Region ausschließlich deshalb zugute, weil sie Hochschulen vorhält. Die Entstehung der direkten Effekte ist dabei unabhängig vom Output der Hochschulen, das heißt, sie fielen auch dann an, wenn alle erbrachten Leistungen der Hochschulen außerhalb des Landes genutzt würden. ${ }^{1}$
Ausgangspunkt der methodischen Überlegungen ist eine Situation, in der das Land vollständig darauf verzichtet, Hochschulen vorzuhalten, so dass der Ressourcenzufluss gleich null ist. Diese Situation wird mit empirisch ermittelten Ist-Werten verglichen. Vier Hauptquellen für Ressourcenzuflüsse lassen sich identifizieren:

a) Einkommen von Studierenden, die außerhalb Sachsen-Anhalts ihre Hochschulzugangsberechtigung (HZB) erworben haben: Diese Einkommen fließen dem Land als Ressource zu, werden zu einem hohen Teil im Land verwendet und führen damit zu Nachfrage, die ohne Hochschulfinanzierung nicht vorhanden wäre.

b) Einkommen der Studierenden mit Elternwohnsitz in Sachsen-Anhalt: Diese Einkommen sind ebenfalls zu berücksichtigen, weil dann, wenn es keine Hochschulen in Sachsen-Anhalt gäbe, diese Studierenden das Land verlassen und die entsprechenden Einkommen anderen Regionen zufließen würden.

c) Forschungsförderungsmittel, die nicht aus dem Landeshaushalt finanziert werden: Diese Drittmittel fließen dem Land zu, werden zum Teil im Land ausgabewirksam und schaffen damit Nachfrage, die ohne Hochschulen dem Land nicht zur Verfügung steht.

d) Bundes- und EU-Mittel, die zweckgebunden dem Hochschulbereich zugewiesen werden: Diese Ressourcen fließen dem Land nur dann zu, wenn es entsprechende Vorleistungen erbringt, d.h. Hochschulen unterhält.

Zur Vereinfachung der Darstellung fassen wir im Folgenden die Positionen a) und b) zusammen zu Studentischen Budgets und die Positionen c) und d) zu Drittmitteln, wobei sich letztere in Ausgaben für Drittmittelbeschäftigte, Sachmittel und Investitionen aufteilen.

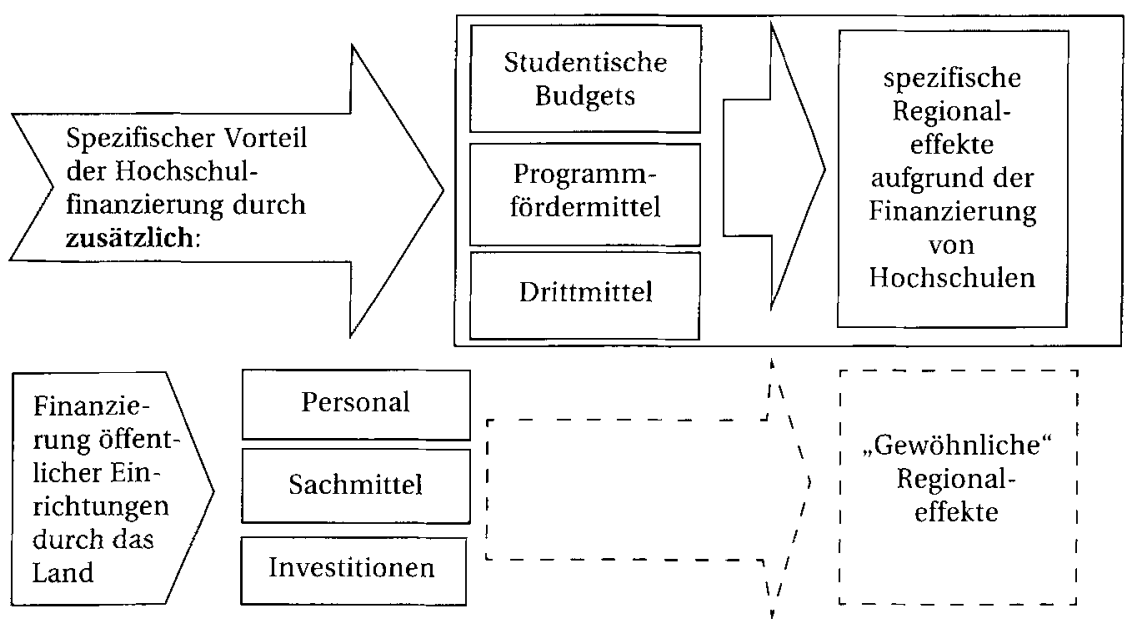

Abbildung 1

Zusätzliche Effekte für das Land aufgrund der Hochschulfinanzierung 
Abbildung 2

Ablauf zur Bestimmung der sektoral und regional disagregierten Nachfrageeffekte durch die Hochschulen

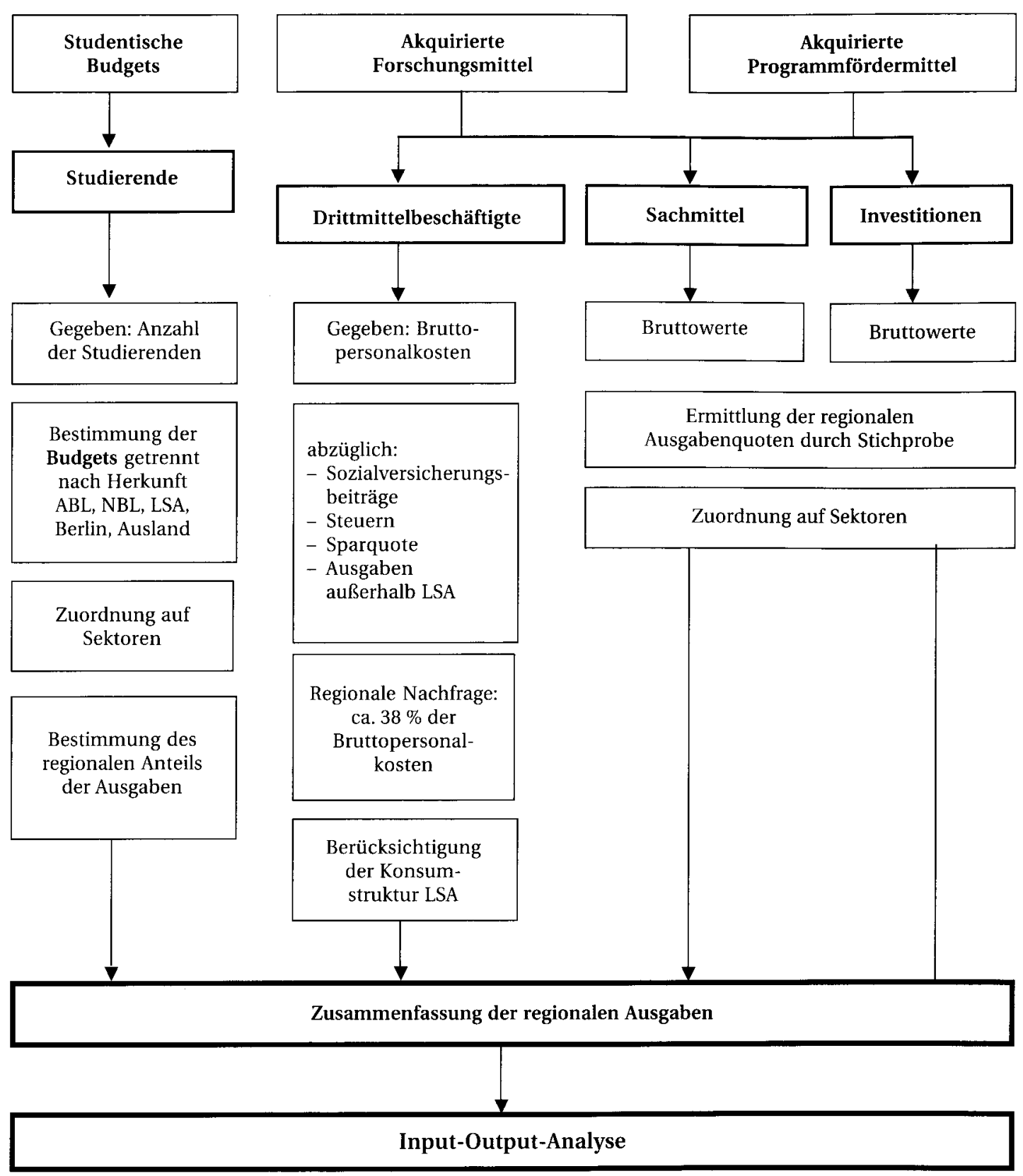

Quelle: eigene Darstellung

Die direkten Effekte der Hochschulfinanzierung werden am Beispiel der Otto-von-Guericke-Universität Magdeburg (OvGU) ${ }^{2}$ und der Hochschule MagdeburgStendal (FH) für die Jahre 1996-2001 untersucht. Vom sehr kleinen FH-Standort Stendal ${ }^{3}$ abgesehen, befinden sich beide Hochschulen in Magdeburg und somit mitten in Sachsen-Anhalt. Da der aus den Hochschulen erwachsende Nutzen vor allem aus der Sicht des Landes interessant ist, das die Hochschulen finanziert, wurde das gesamte Bundesland als Bezugs- und Un- tersuchungsregion gewählt. Das aufwändige Vorgehen zur Bestimmung der regionalen und sektoralen Inzidenz der Ausgaben von Studierenden und von Drittmitteln erfolgt weitgehend in Anlehnung an Blume/ Fromm (1999) und ist ausführlich in Rosner / Weimann (2003) dargestellt. Die wichtigsten Schritte fasst Abbildung 2 zusammen.

Die sektoral und regional differenzierten Ausgabepositionen bilden den Ausgangspunkt für die weitere Untersuchung der regionalökonomischen Wirkungen im 
Rahmen einer offenen Input-Output-Analyse. ${ }^{4}$ Unsere Berechnungen stützen sich auf die Input-OutputTabelle für das Jahr 2000 des Statistischen Bundesamtes (2002), die für Sachsen-Anhalt unter äußerst konservativen Annahmen regionalisiert wurde (Rosner/ Weimann 2003, S. 50-52).

Insgesamt können mit der Input-Output-Analyse Umsatz-, Wertschöpfungs- und Einkommenseffekte ermittelt werden. Die Bestimmung der Beschäftigungseffekte erfolgt rekursiv auf Grundlage sektoral differenzierter Umsatzmultiplikatoren.

Die Umsatzeffekte geben sämtliche Umsätze an, die aufgrund von Mittelzuflüssen durch die Hochschulen nach Sachsen-Anhalt entstehen. In regionalökonomischen Untersuchungen werden sie oft als zentrale GröBe dargestellt. Gleichwohl greift die reine Betrachtung von Umsätzen zu kurz, denn Umsätze führen erst nach Abzug von Vorleistungsbezügen, Importen und Steuern zu Wertschöpfung - und in der Folge zu Einkommen. Vor diesem Hintergrund wurden auch Einkommens- und Wertschöpfungseffekte ermittelt.

Die Wertschöpfungseffekte geben den Teil der Umsätze an, der nach Abzug von Vorleistungen, Steuern und Importen verbleibt. Volkswirtschaftlich ist die Wertschöpfung der bedeutendste Indikator, da er widerspiegelt, in welchem Umfang neue Werte geschaffen wurden. Verwandt wird die Wertschöpfung für die Entlohnung der Produktionsfaktoren, also für Löhne und Gehälter, für Kapitalentlohnung und für Unternehmerlohn. Grundsätzlich entsteht durch die Wertschöpfung also Einkommen.

Die Einkommenseffekte weichen in der vorliegenden Untersuchung aus zwei Gründen von der Wertschöpfung ab: Erstens wurde unterstellt, dass die Drittmittelbeschäftigten (wie die Hochschulen insgesamt) nichts produzieren, weshalb ihre Arbeitsleistung nicht in den Wertschöpfungs-, wohl aber in den Einkommenseffekten berücksichtigt ist. Zweitens wurde der Arbeitgeberanteil an den Beiträgen zur Sozialversicherung von der Wertschöpfung abgezogen, um die Einkommenseffekte (hier definiert als Bruttoeinkommen der Haushalte) zu bemessen. Hat eine Hochschule besonders viele Drittmittelbeschäftigte, so übersteigen die Einkommenseffekte die Wertschöpfungseffekte, denn die extern finanzierten Drittmittelbeschäftigten tragen zwar zur Erhöhung der Einkommenseffekte, nicht jedoch zu den Wertschöpfungseffekten bei. Erbringt die Hochschule dagegen vor allem Leistungen der Lehre (insbesondere Fachhochschulen), so können die Wertschöpfungseffekte die Einkommenseffekte übersteigen.
Schließlich werden die von den Hochschulen ausgehenden Beschäftigungseffekte bestimmt. Für den regionalen Arbeitsmarkt sind Hochschulen oft von großer Bedeutung. Diese Bedeutung entsteht wesentlich durch die direkten Beschäftigungseffekte, d.h. durch die Arbeitgeberrolle der Hochschulen. Grundsätzlich hätte ein Land als Zuwendungsgeber aber auch die Möglichkeit, Personal für andere Zwecke anzustellen, wodurch sich ähnliche Arbeitsplatzeffekte ergeben würden. Vor diesem Hintergrund werden nur solche direkten Beschäftigungseffekte untersucht, die auf Mittelzuflüsse von außerhalb des Landes zurückgeführt werden können. Dadurch wird der spezifische Vorteil der Hochschulfinanzierung gegenüber alternativen Ausgabemöglichkeiten des Landes deutlich.

Nach der Bestimmung der Umsatz- Wertschöpfungs-, Einkommens- und Beschäftigungseffekte der Hochschulen werden diese ins Verhältnis zu den Aufwendungen des Landes gesetzt. Die so berechneten regionalen Rates of Return der Hochschulfinanzierung ermöglichen politischen Entscheidungsträgern einen direkten Vergleich alternativer Mittelverwendungsmöglichkeiten.

\section{Mittelzuflüsse und regionalökonomische Effekte durch die Hochschulen im nördlichen Teil Sachsen-Anhalts}

Empirisch wurden die regionalen Rates of Return am Beispiel der Otto-von-Guericke-Universität Magdeburg (OvGU) und der Hochschule Magdeburg-Stendal $\mathrm{FH})$ berechnet. Im Folgenden werden zunächst die regionalwirtschaftlichen Effekte, die zwischen 1996 und 2001 auf diese Mittelzuflüsse zurückzuführen sind, für beide Hochschulen zusammen dargestellt. Es lassen sich strukturelle Unterschiede zwischen Universität und Fachhochschule ausmachen, die im Wesentlichen darauf zurückzuführen sind, dass Fachhochschulen vor allem Lehraufgaben haben, während Universitäten Lehre und Forschung abdecken. ${ }^{5}$

Allein aufgrund der Existenz von OvGU und FH wurden Ressourcenzuflüsse nach Sachsen-Anhalt (LSA) ausgelöst, die sich von 144,6 Mio. DM in $1996 \mathrm{um}$ 94,2 Mio. DM auf 238,8 Mio. DM in 2001 erhöht haben (Tab. 1). Dabei ist der kontinuierliche Zuwachs um insgesamt $65 \%$ vor allem auf die stark gestiegenen Studierendenzahlen zurückzuführen.

Von den Mittelzuflüssen des Jahres 2001 entfallen $7 \%$ (170,5 Mio. DM) auf die OvGU und $29 \%$ (68,3 Mio. DM) auf die FH. Infolge der gestiegenen Studierendenzahlen konnte die FH die von ihr ausgelösten Mittelzuflüsse verdoppeln; die OvGU verzeichnete einen 
Tabelle 1

Mittelzuflüsse nach Sachsen-Anhalt durch die Otto-von-Guericke-Universität Magdeburg und die Hochschule Magdeburg-Stendal (FH) (in DM)

\begin{tabular}{|l|r|r|r|r|r|r|}
\hline Mittelzuflüsse & $\mathbf{1 9 9 6}$ & $\mathbf{1 9 9 7}$ & $\mathbf{1 9 9 8}$ & $\mathbf{1 9 9 9}$ & $\mathbf{2 0 0 0}$ & $\mathbf{2 0 0 1}$ \\
\hline Studierende & & & & & \\
Budgets & 97088242 & 107233713 & 121.109336 & 144333543 & 165543324 & 183173079 \\
Summe reg. Ausgaben & 76354899 & 84940280 & 95936493 & 114374922 & 130806209 & 143011676 \\
\hline Drittmittel-Beschäftigte & & & & & \\
Personalkosten & 18865119 & 18001991 & 17814342 & 19878665 & 20664098 & 23846802 \\
Bruttoeinkommen & 15673097 & 14863552 & 14695271 & 16440878 & 17126599 & 19792341 \\
Summe reg. Konsumausgaben & 7286723 & 6829319 & 6740230 & 7578589 & 7926477 & 9184655 \\
\hline Sachmittel & & & & & & \\
Sachmittelausgaben gesamt & 12503226 & 9160347 & 8792732 & 6947863 & 6.925 .547 & 7976144 \\
regional wirksame Nachfrage & 3610932 & 2645508 & 2539341 & 2006542 & 2.000 .098 & 2303510 \\
\hline Investitionen & & & & & & \\
Investitionen gesamt & 16140207 & 19703751 & 17544912 & 17487736 & 19642306 & 23758534 \\
Summe der reg. Ausgaben & 11503794 & 14289310 & 12692833 & 12482378 & 13570859 & 15807825 \\
\hline Gesamt & & & & & & \\
Mittelzuflüsse nach LSA & 144596794 & 154099802 & 165261322 & 188647807 & 212775275 & 238754560 \\
Summe der reg. Ausgaben & 98756347 & 108704418 & 117908897 & 136442432 & 154303643 & $\mathbf{1 7 0 3 0 7 6 6 6}$ \\
\hline
\end{tabular}

Quelle der Daten:

Otto-von-Guericke-Universität Magdeburg, Hochschule Magdeburg-Stendal (FH), Staatshochbauamt Magdeburg, Landesregierung Sachsen-Anhalt, Deutsches Studentenwerk, eigene Berechnungen

Tabelle 2

Nachfrageeffekte, Wertschöpfung und Einkommen der Haushalte in Sachsen-Anhalt durch Studierende, Drittmittelbeschäftigte und Verausgabung extern eingeworbener Mittel an der Otto-von-Guericke-Universität Magdeburg und der Hochschule MagdeburgStendal (in DM)

\begin{tabular}{|l|r|r|r|r|r|r|}
\hline & $\mathbf{1 9 9 6}$ & $\mathbf{1 9 9 7}$ & $\mathbf{1 9 9 8}$ & $\mathbf{1 9 9 9}$ & $\mathbf{2 0 0 0}$ & $\mathbf{2 0 0 1}$ \\
\hline Mittelzuflüsse nach LSA & 144596794 & 154099802 & 165261322 & 188647807 & 212775275 & 238754560 \\
\hline Summe der reg. Ausgaben & 98756347 & 108704418 & 117908897 & 136442432 & 154303643 & 170307666 \\
\hline $\begin{array}{l}\text { Summe der direkten, indirekten } \\
\text { und induzierten Umsatzeffekte }\end{array}$ & 183575863 & 163756739 & 177688054 & 205797780 & 233552200 & 258174944 \\
\hline Wertschöpfung in LSA & 69486860 & 76040667 & 82690248 & 95530936 & 108719783 & 120238948 \\
\hline Bruttoeinkommen der Haushalte & 79332215 & 83713229 & 89332629 & 102418571 & 114645800 & 127796149 \\
\hline
\end{tabular}

Quelle: eigene Berechnungen

Zuwachs um $65 \%$. Die größeren Wachstumsraten der FH sind dadurch zu erklären, dass es sich um eine neu gegründete Fachhochschule handelt, die im Gegensatz zur Universität noch nicht auf einem bestehenden Sockel aufbauen konnte.

Die Mittelzuflüsse des Jahres 2001 führten zu regional wirksamer Nachfrage im Umfang von 170,3 Mio. DM. Diese Nachfrage geht zu $84 \%$ auf die Studierenden zurück. Die regionalen Ausgaben aus externen Mittelzuflüssen sind bei der $\mathrm{FH}$ zu $93 \%$ auf die Studierenden zurückzuführen, während der Vergleichswert der OvGU bei $80 \%$ liegt. Die Differenz ergibt sich aus der Tatsache, dass die FH hauptsächlich Lehrleistungen erbringt, während die Universität Lehr- und Forschungsleistungen produziert. Entsprechend war sie auch erfolgreicher bei der Einwerbung von forschungsbezogenen Drittmitteln.

Durch die zusätzlich attrahierten Mittelzuflüsse in 2001 bewirkten die beiden Hochschulen Umsätze in Sachsen-Anhalt in Höhe von 258,2 Mio. DM. Daraus resultierte eine Wertschöpfung in Höhe von 120,2 Mio. DM und ein Beitrag zum Einkommen der privaten Haushalte in Höhe von 127,8 Mio. DM (Tab. 2). Der rasante Anstieg der regionalökonomischen Effekte ist eng mit der Zunahme der Studierendenzahlen verbunden. 
Tabelle 3

Sektorale Verteilung der direkten, indirekten und induzierten Nachfrageeffekte durch Studierende und Drittmittelausgaben der Otto-von-Guericke-Universität Magdeburg und der Hochschule Magdeburg-Stendal (in DM)

\begin{tabular}{|c|c|c|c|c|c|c|}
\hline Wirtschaftsabteilung & 1996 & 1997 & 1998 & 1999 & 2000 & 2001 \\
\hline A Land- und Forstwirtschaft & 1669250 & 1815323 & 1999607 & 2361044 & 2683491 & 2954371 \\
\hline B Fischerei und Fischzucht & 14925 & 16320 & 18013 & 21333 & 24224 & 26607 \\
\hline $\begin{array}{l}\text { C Bergbau, Gewinnung von } \\
\text { Steinen und Erden }\end{array}$ & 1411437 & 1547014 & 1708444 & 2015946 & 2296822 & 2525521 \\
\hline D Verarbeitendes Gewerbe & 30469409 & 33952875 & 36483829 & 43753608 & 48326457 & 52724177 \\
\hline E Energie- und Wasserversorgung & 5898708 & 6478472 & 7220644 & 8545833 & 9775477 & 10729390 \\
\hline F Baugewerbe & 9602052 & 9121541 & 9219653 & 9110554 & 11255137 & 13736899 \\
\hline G Handel, Instandhaltung, Reparatur & 22867824 & 26425726 & 27960539 & 30644117 & 34653896 & 38182732 \\
\hline H Gastgewerbe & 3639565 & 3961131 & 4378550 & 5160378 & 5878128 & 6473898 \\
\hline I Verkehr, Nachrichtenübermittlung & 16974751 & 18569678 & 20630361 & 24329225 & 27603143 & 30374713 \\
\hline I Kredit- und Versicherungsgewerbe & 4410563 & 4830308 & 5351531 & 6295335 & 7191271 & 7912464 \\
\hline $\begin{array}{l}\text { K Grundstücks- und Wohnungswesen, } \\
\text { Vermietung, unternehmensnahe } \\
\text { Dienstleistungen } \\
\end{array}$ & 40144300 & 43485909 & 47692999 & 55876276 & 63698827 & 70355423 \\
\hline $\begin{array}{l}\text { Öffentliche Verwaltung, } \\
\text { Verteidigung, Sozialversicherung }\end{array}$ & 643681 & 677892 & 741346 & 852423 & 966429 & 1066059 \\
\hline M Erziehung und Unterricht & 704324 & 768654 & 853568 & 1009561 & 1149960 & 1264425 \\
\hline N Gesundheits-, Veterinär- u. Sozialwesen & 1048323 & 1127450 & 1228817 & 1439410 & 1623837 & 1804102 \\
\hline $\begin{array}{l}\text { O Sonstige öffentliche und } \\
\text { persönliche Dienstleistungen }\end{array}$ & 9570114 & 10479134 & 11642976 & 13722538 & 15673084 & 17217581 \\
\hline$P$ Dienstleistungen priv. Haushalte & 454028 & 499315 & 557176 & 660199 & 752017 & 826581 \\
\hline Gesamt & 149523253 & 163756739 & 177688054 & 205797780 & 233552200 & 258174944 \\
\hline
\end{tabular}

Quelle: eigene Berechnungen

Tabelle 4

Branchenspezifische direkte, indirekte und induzierte Beschäftigungseffekte der Otto-von-Guericke-Universität Magdeburg und der Hochschule Magdeburg-Stendal (FH)

\begin{tabular}{|c|c|c|c|c|c|c|}
\hline Sektor & 1996 & 1997 & 1998 & 1999 & 2000 & 2001 \\
\hline A Land- und Forstwirtschaft & 8 & 9 & 9 & 10 & 12 & 13 \\
\hline B Fischerei und Fischzucht & 0 & 0 & 0 & 0 & 0 & 0 \\
\hline C Bergbau, Gewinnung von Steinen und Erden & 3 & 3 & 3 & 3 & 4 & 4 \\
\hline D Verarbeitendes Gewerbe & 103 & 112 & 119 & 140 & 151 & 162 \\
\hline E Energie- und Wasserversorgung & 21 & 22 & 25 & 28 & 32 & 35 \\
\hline F Baugewerbe & 106 & 99 & 98 & 96 & 116 & 138 \\
\hline G Handel, Instandhaltung, Reparatur & 266 & 302 & 313 & 336 & 373 & 403 \\
\hline $\mathrm{H}$ Gastgewerbe & 79 & 85 & 92 & 106 & 118 & 127 \\
\hline I Verkehr, Nachrichtenübermittlung & 105 & 113 & 123 & 143 & 159 & 171 \\
\hline J Kredit- und Versicherungsgewerbe & 23 & 25 & 27 & 31 & 35 & 38 \\
\hline $\begin{array}{l}\text { K Grundstücks- und Wohnungswesen, Vermietung, } \\
\text { unternehmensnahe Dienstleistungen }\end{array}$ & 196 & 208 & 224 & 257 & 287 & 311 \\
\hline L Öffentliche Verwaltung, Verteidigung, Sozialversicherung & 6 & 7 & 7 & 7 & 9 & 10 \\
\hline M Erziehung und Unterricht & 282 & 282 & 291 & 320 & 299 & 340 \\
\hline N Gesundheits-, Veterinär- und Sozialwesen & 14 & 15 & 16 & 19 & 20 & 23 \\
\hline O Sonstige öffentliche und persönliche Dienstleistungen & 90 & 96 & 105 & 121 & 135 & 146 \\
\hline $\mathrm{P}$ Dienstleistungen privater Haushalte & 48 & 51 & 56 & 65 & 72 & 78 \\
\hline Gesamt & 1352 & 1429 & 1507 & 1683 & 1824 & 1998 \\
\hline
\end{tabular}

Quelle: eigene Berechnungen 
Die sektorale Gliederung der Umsatzeffekte zeigt Tabelle 3. Dabei fällt auf, dass der Sektor Grundstücks- und Wohnungswesen, Vermietung und Erbringung von überwiegend unternehmensnahen Dienstleistungen mit 70,4 Mio. DM in 2001 besonders stark von der Existenz der Hochschulen profitiert. In diesem Sektor sind die Mietzahlungen der Studierenden und der Drittmittelbeschäftigten enthalten, die vorrangig in der Stadt Magdeburg anfallen. Allein diese Zahl verdeutlicht die Bedeutung der Hochschulen für den regionalen Wohnungsmarkt. Zwischen den beiden Hochschulen sind bei der sektoralen Verteilung der Umsatzeffekte kaum Unterschiede festzustellen, denn sie gehen vorrangig auf die Studierenden zurück, deren Konsumstruktur durch die Wahl der Hochschule nicht beeinflusst ist.

Die Beschäftigungseffekte aufgrund von Mittelzuflüssen nach Sachsen-Anhalt sind eng mit den Umsatzeffekten verbunden (Tab. 4). In 2001 wird der Wohnungssektor nur vom Bereich Handel, Instandhaltung und Reparatur von KfZ und Gebrauchsgütern übertroffen, in dem 403 Beschäftige auf die Existenz der Hochschulen zurückzuführen sind. Der besonders hohe Wert von 340 Beschäftigten im Bereich Erziehung und Unterricht erklärt sich durch die 325 direkt Drittmittel- beschäftigten aus externen Mittelzuflüssen, von denen die überwiegende Zahl (291) an der OvGU arbeitet (FH: 34).

\section{Aufwendungen des Landes und die regionalen Rates of Return der Hochschulfinanzierung}

Den Effekten der Hochschulen sind die Aufwendungen des Landes gegenüber zu stellen (Abb. 3). Für beide Hochschulen wurden in 1996166,8 Mio. DM aufgewandt, während es in 2000 188,4 Mio. DM waren. Die Steigerung der Gesamtaufwendungen des Landes um $13 \%$ entfällt fast vollständig auf die im Aufbau befindliche FH, deren Haushaltsmittel im Betrachtungszeitraum kontinuierlich um insgesamt $77 \%$ auf 42,7 Mio. DM anwuchsen. Unterdessen verzeichnet die OvGU seit 1998 einen jährlichen Rückgang der Landeszuweisungen, so dass das nominale Budget des Jahres 2001 nur noch $2 \%$ über dem Ausgangswert des Jahres 1996 liegt. Der Rückgang der Mittelzuweisungen an die OvGU ist in erster Linie auf das gesunkene Investitionsvolumen zurückzuführen, während sich die anderen Ausgabepositionen konstant entwickelten.

\section{Abbildung 3}

Aufwendungen des Landes für die Otto-von-Guericke-Universität Magdeburg und die Hochschule Magdeburg-Stendal (FH), Mittelzuflüsse in das Land durch die Hochschulen und daraus entstehende regionale Effekte (in DM)

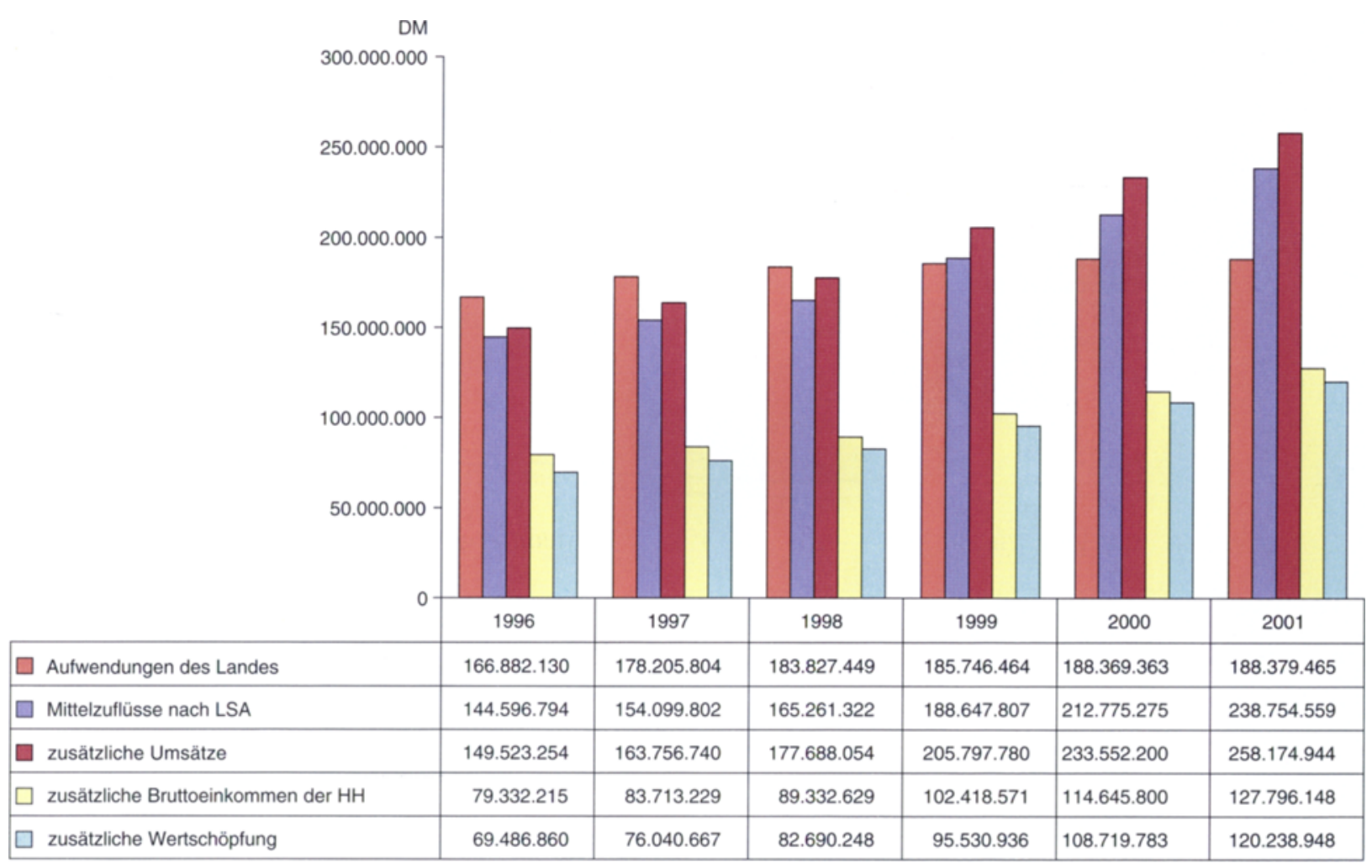

Quelle: eigene Berechnungen 
Abbildung 3 zeigt neben den Aufwendungen des Landes auch die daraus resultierenden zusätzlichen regionalwirtschaftlichen Effekte im Zeitablauf. Die aus eigener Kraft der Hochschulen generierten Mittelzuflüsse nach Sachsen-Anhalt sind seit dem Jahr 1999 größer als die Aufwendungen des Landes für die Hochschulen. Parallel dazu sind auch die Wertschöpfungsund Einkommenseffekte angestiegen. Die Ergebnisse verdeutlichen, dass Hochschulen in der Tat handelbare Güter produzieren.

Wie verhalten sich die berechneten regionalwirtschaftlichen Effekte in Relation zu den Mittelaufwendungen des Landes? Dieses Verhältnis wird durch die regionalen Rates of Return der Hochschulfinanzierung abgebildet (Abb. 4).

Im Ergebnis zeigt sich, dass eine DM, die in 2001 vom Land in die Otto-von-Guericke-Universität Magdeburg und in die Hochschule Magdeburg-Stendal (FH) investiert wurde, einen zusätzlichen Mittelzufluss nach Sachsen-Anhalt in Höhe von 1,27 DM bewirkte. Lediglich aus diesen Zuflüssen entstanden im Land:

- Umsätze in Höhe von 1,37 DM,

- Bruttoeinkommen der privaten Haushalte in Höhe von $0,68 \mathrm{DM}$ und

- Wertschöpfung in Höhe von 0,64 DM.
Diese Werte verdeutlichen die Nachfrageeffekte, die durch die Hochschulfinanzierung induziert werden. Sie liegen deutlich über den Mittelaufwendungen des finanzierenden Landes zum Unterhalt der Einrichtungen und verdeutlichen damit, dass öffentliche Wissenschaftseinrichtungen aus regionalwirtschaftlicher Sicht besonders rentable Investitionen darstellen. Selbst wenn alle Absolventen nach ihrem Studium die Region verlassen, die Hochschule keine Forschungsergebnisse produziert und keinen Wissenstransfer in die (regionale) Wirtschaft leistet, wären die Ergebnisse von diesem Umstand nicht direkt betroffen, da Humankapital- und Kapazitätseffekte in der vorliegenden Berechnung unberücksichtigt bleiben. Die zusätzliche Betrachtung solcher Effekte würde die regionalen Rates of Return weiter deutlich erhöhen. Die Resultate legen nahe, dass Hochschulen eine besonders hohe Selbstfinanzierungsquote aufweisen.

Da die Fachhochschule hauptsächlich Lehrleistungen erbringt, die besonders starke direkte regionalwirtschaftliche Effekte nach sich ziehen, liegen ihre regionalen Rates of Return deutlich über denen der OvGU. Gleichwohl kann daraus nicht gefolgert werden, dass Fachhochschulen insgesamt produktiver sind, da die Forschungsleistungen, die in wesentlich stärkerem Maße von der Universität erbracht werden, nicht in die

Abbildung 4

Regionale Rates of Return des Landes für die Otto-von-Guericke-Universität Magdeburg und die Hochschule MagdeburgStendal (FH). Zusätzliche regionalökonomische Effekte je eingesetzter Geldeinheit:

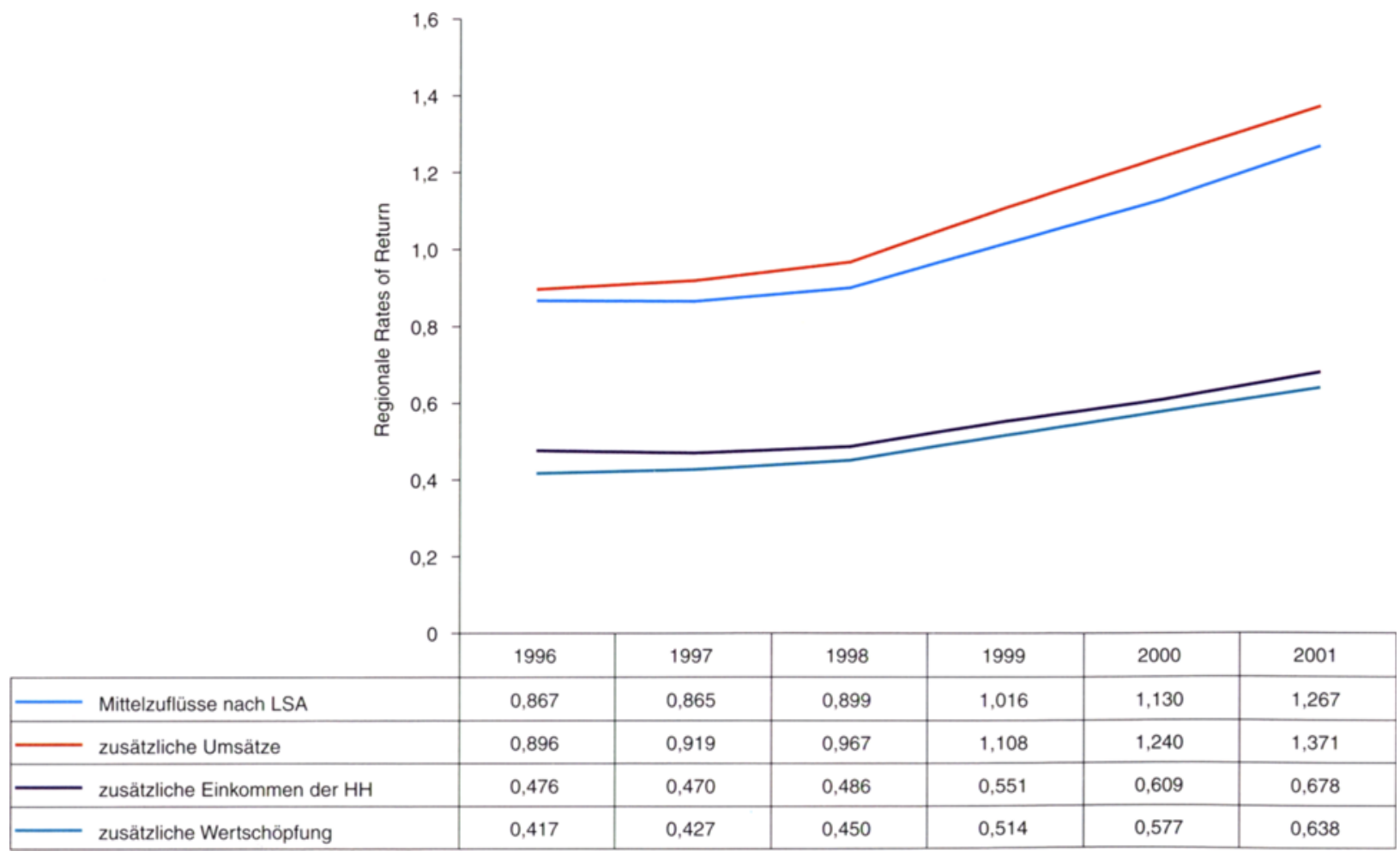

Quelle: eigene Berechnungen 
Berechnungen eingehen. Gerade Forschungsleistungen sind notwendige Bedingung für weitere erhebliche Mittelzuflüsse in die Region, die vor allem durch die Ansiedlung weiterer Wissenschaftseinrichtungen entstehen, beispielsweise Max-Planck- oder FraunhoferInstitute.

Die regionalwirtschaftlichen Effekte der Hochschulen werden in erster Linie durch die Studierenden hervorgerufen, da sie den überwiegenden Teil der Mittelzuflüsse generieren. Bei alleiniger Betrachtung der studentischen Ausgaben im Rahmen der InputOutput-Analyse zeigt sich, dass zehn zusätzliche Studierende eine Zunahme um einen Beschäftigten in Sachsen-Anhalt bewirken. Ergänzend attrahieren die Hochschulen Ressourcenzuflüsse durch die Programm- und Forschungsförderung, wodurch ebenfalls Beschäftigungseffekte im Land entstehen, ohne dass damit Kosten für den Landeshaushalt verbunden sind.

Die Relation aus allen Beschäftigungseffekten, die auf zusätzliche Mittelzuflüsse zurückzuführen sind, und aus den Aufwendungen des Landes zeigt, dass die beiden Hochschulen in 2001 je investierte $94284 \mathrm{DM}$ einen zusätzlichen Arbeitsplatz geschaffen haben. ${ }^{6}$ Darin nicht einbezogen sind die ca. 1700 direkt Beschäftigten der beiden Hochschulen aus Landesmitteln. Rechnet man diese noch hinzu, so belaufen sich die Kosten eines Arbeitsplatzes, der durch die Hochschulen geschaffen wird, auf lediglich 50941 DM pro Jahr, ohne dass dabei berücksichtigt wäre, dass die Hochschulen Humankapital produzieren, über Ausgründungen Arbeitsplätze schaffen, durch Wissenstransfer zur Steigerung der Wettbewerbsfähigkeit der (regionalen) Unternehmen und zur Ansiedlung von Forschungsinstituten beitragen.

\section{Fazit}

Drei wesentliche Innovationen für die Untersuchung der regionalwirtschaftlichen Effekte von Hochschulen werden in diesem Beitrag vorgestellt: die regionalen Rates of Return der öffentlichen Hochschulfinanzierung, die empirische Untersuchung von Hochschulen über einen längeren Zeitraum und der direkte Vergleich einer Universität mit einer Fachhochschule.

Mit den regionalen Rates of Return bemessen wir den spezifischen regionalökonomischen Vorteil der öffentlichen Finanzierung von Hochschulen. Dies geschieht, indem die Aufwendungen des Landes ins Verhältnis zu den Effekten gebracht werden, die aus zusätzlich attrahierten Mittelzuflüssen in die Region entstehen. Mit dieser Methode wird gezeigt, dass Hochschulen aus eigener Kraft größere regionalökonomische Effekte er- zielen, als Mittel zu ihrer Finanzierung notwendig sind. Gleichzeitig bietet sie die Voraussetzung zum Vergleich alternativer Projekte der öffentlichen Hand, sodass Investitionsentscheidungen optimiert werden können. Darüber hinaus zeigen die empirischen Ergebnisse, dass Investitionen im Hochschulbereich eine besonders effektive und kostengünstige Form der Wirtschafts- und Arbeitsmarktpolitik darstellen. Vor allem mit Blick auf den wirtschaftlichen Aufholprozess der neuen Bundesländer ist diese Erkenntnis von größter Bedeutung. Die Frage, ob Hochschulen bei gleichem Mitteleinsatz größere Effekte hervorrufen als Förderprogramme, muss an dieser Stelle der weiteren Forschung überlassen bleiben.

Durch den Untersuchungszeitraum über sechs Jahre wird deutlich, dass Hochschulen in der Aufbauphase kontinuierlich zunehmende Effekte aufweisen. Trotz kaum gestiegener Aufwendungen des Landes konnten zunehmend mehr Studierende und Drittmittel in die Region gezogen werden. Offenkundig benötigen Hochschulen im Aufbauprozess Zeit, um ihre regionalökonomische Wirksamkeit voll entfalten zu können. Diese steigt kontinuierlich mit der Zahl der Studierenden und dem Volumen der eingeworbenen Drittmittel.

Erstmalig werden eine Universität und eine Fachhochschule mit derselben Methode untersucht, wodurch strukturelle Unterschiede in der regionalökonomischen Wirkungsweise deutlich werden. Im Vergleich zeigt sich, dass die Fachhochschule in Relation zum Mitteleinsatz größere direkte Effekte als die Universität generiert. Da indirekte Effekte (z.B. Ansiedlung von Unternehmen und Forschungsinstituten, Ausgründungen, Forschungskooperationen) in dieser Untersuchung unberücksichtigt bleiben, darf daraus jedoch nicht der Schluss gezogen werden, dass Fachhochschulen grundsätzlich eine größere regionalökonomische Bedeutung haben. Gerade die indirekten Effekte, die in starkem Maße angebotsseitig wirken, werden vorrangig von Universitäten produziert.

Insgesamt zeigt die Untersuchung, dass Sparmaßnahmen im Hochschulsektor nicht nur langfristig negative Folgen für eine Region haben können, weil sie zu einer schlechteren Humankapitalversorgung und geringeren Wissenstransfers führen, sondern auch kurzfristig erhebliche Opportunitätskosten verursachen.

\section{Anmerkungen}

* Die Arbeit entstand im Rahmen des Forschungsprojekts "Ökonomische Effekte der Hochschulausgaben des Landes Sachsen-Anhalt", das vom Kultusministerium Sachsen-Anhalt gefördert wird. 
(1)

Man stelle sich vor, dass alle Absolventen das Land verlassen und keinerlei verwertbare Forschungsergebnisse erzielt werden.

(2)

Die Medizinische Fakultät der Otto-von-Guericke-Universität mit dem Universitätsklinikum wurde in der Untersuchung nicht berücksichtigt.

(3)

Auf den Standort Stendal entfallen unter $7 \%$ der Studierenden der gesamten Hochschule.

(4)

Zum Verfahren der Input-Output-Analyse vgl. z.B. Holub/ Schnabl (1985), Clermont (1997), Strassert (1968), Niermann (1996), Fleissner et al. (1993)

(5)

Die detaillierten Einzelergebnisse für die beiden untersuchten Hochschulen können gerne per Email bei den Autoren angefordert werden.

(6)

Der Wert errechnet sich aus den eingesetzten Mitteln des Landes im Verhältnis zu den Beschäftigungseffekten aus extern attrahierten Mitteln der Hochschulen. Bei der FH lag dieser Wert bei $81.747 \mathrm{DM}$, bei der OvGU bei $101352 \mathrm{DM}$.

\section{Literatur}

Blume, L.; Fromm, O. (1999): Regionale Ausgabeneffekte von Hochschulen: Methodische Anmerkungen am Beispiel der Universität Gesamthochschule Kassel. In: Raumforschung und Raumordnung 5-6/1999, S. 418-431

Blume, L.; Fromm, O. (2000): Regionalökonomische Bedeutung von Hochschulen: Eine empirische Untersuchung am Beispiel der Universität Gesamthochschule Kassel. - Wiesbaden

Clermont, C. (1997): Regionalwirtschaftliche Effekte von Wissenschaftseinrichtungen - Theorie, Messkonzepte und Ergebnisse für Hamburg. - Frankfurt/M. u.a.

Fleissner, P.; Böhme, W.; Brautzsch, H.-U.; Höhne, J.; Siassi, J.; Stark, K. (1993): Input-Output-Analyse: Eine Einführung in Theorie und Anwendungen. - Wien und New York
Gloede, K.; Schirmag, T.; Schöler, K. (1999): Ökonomische Wirkungen der Universität Potsdam auf die Region. - Frankfurt/M. u.a.

Holub H.-W.; Schnabel, H. (1985): Input-Output-Rechnung: Input-Output-Tabellen. - München und Wien

Leontief, W. (1936): Quantitative Input and Output Relations in the Economic System of the United States. In: Review of Economic Statistics, Vol. 18, No. 3, S. 105-125

Niermann, U. (1996): Wirtschaftsfaktor Universität: Eine InputOutput-orientierte Analyse am Beispiel der Universität Bielefeld. - Münster

Pfähler, W.; Bönte, W.; Gabriel, C.; Kettner, A. (1999): Wirtschaftsfaktor Bildung und Wissenschaft: Die regionalwirtschaftliche Bedeutung der Hochschulbildungs- und Wissenschaftseinrichtungen in Bremen. - Frankfurt/M. u.a.

Rosner, U.; Weimann, J. (2003): Die ökonomischen Effekte der Hochschulausgaben des Landes Sachsen-Anhalt - Direkte monetäre Effekte der Otto-von-Guericke-Universität Magdeburg und der Hochschule Magdeburg-Stendal (FH) (Teil 1), FEMM Working Paper Nr. 12/2003. - Magdeburg

Statistisches Bundesamt (2002): Volkswirtschaftliche Gesamtrechnungen, Input-Output-Tabellen. - Wiesbaden

Strassert, G. (1968): Möglichkeiten und Grenzen der Erstellung und Auswertung regionaler Input-Output-Tabellen unter besonderer Berücksichtigung der derivativen Methode. - Berlin

Dipl.-Vw. Ulf Rosner, M.A.

Prof. Dr. Joachim Weimann

Otto-von-Guericke-Universität Magdeburg

Fakultät für Wirtschaftswissenschaft, VWL III

Postfach 4120

39016 Magdeburg

E-Mail: ulf.rosner@ww.uni-magdeburg.de

E-Mail: joachim.weimann@ww.uni-magdeburg.de 